\title{
Synthesis
}

\section{Infodynamics, a Developmental Framework for Ecology/Economics}

\author{
Stanley N. Salthe ${ }^{1}$
}

\begin{abstract}
Infodynamics, for our purposes, is a developmental perspective that animates information theory by way of thermodynamics. The isomorphism between Boltzmann's statistical interpretation of physical entropy as disorder and Shannon's formulation of variety as informational entropy signals a deep connection between information and entropy production. Information is any configuration that might have been different, providing that it delays energy dissipation so that the energy is dissipated more completely. The entropy production of individual dissipative structures increases at first but eventually decelerates. I consider the questions: why do these structures grow? And why don't they keep on growing? As the universal expansion of the Big Bang accelerated, matter precipitated from disequilibrated energy. In its own search for equilibrium, matter clumped, signaling further disequilibrium. The only way these clumps can be destroyed is by others, and this role of gradient degradation entrained the evolution of complexity, all the way to living systems. This serves universal equilibration because, generally, more of an energy gradient must be lost as heat than can become reembodied in its consumers, and so it can be said that these structures grow to serve gradient degradation, taking the second law of thermodynamics as a final cause. I suggest that energy degradation is harnessed by growing systems because that process allows the fastest eventual dissipation in the direction of the lowest grade of energy. Three stages of development of dissipative structures are described: immature, mature, and senescent. Growth is limited by senescence, which I take to be a consequence of information overload. I suggest that ecosocial systems harnessed by human population growth impose less information on ecological transformations than do typical mature ecosystems, thereby tapping more powerful energy flows and producing more wastes of a higher grade than heat, which act as pollutants. Warfare is interpreted as a mechanism to prevent ecosocial senescence. I suggest that ecosocial systems should be planned in the direction of maintaining system maturity as long as possible.
\end{abstract}

\section{INTRODUCTION}

From the point of view of infodynamics, an ecosystem is an energy transformation system that is increasingly constrained by informational arrays (Jørgensen $2001 b$ ). Whatever happens in the material world, i.e., work such as moving air by winds, is brought about by energy, but most of this energy is dissipated to a lower grade rather than driving that work. That is, the faster any work is done, the more of the energy gradient is converted to forms of energy that are incapable of driving that kind of work, i.e., the energy efficiency of the process drops. Because the most powerful events dissipate most of an energy gradient, only lesser gradients are left to be exploited by weaker forces until the energy has been degraded all the way to heat, at which point it has become so disorganized that it is unable to support any further definite material events other than Brownian motion. This disordering of energy gradients is taken to be their contamination by entropy. The universal drive to contaminate energy gradients in this way is called the second law of thermodynamics, which was activated when the Big Bang burst out of energy equilibrium.

Ecosystems, which include abiotic ones such as those found on the moon, interrupt the degradation of highquality energy such as sunshine by imposing a cascade of increasingly weaker events, each of which represents work, between the production of the energy and its final dissipation to heat. The effect of this at any one locale is to take the dissipation further in the direction of heat at that particular point, that is, to more completely dissipate more of the energy locally. The origin of life resulted in the placement of relatively elaborate, i.e., information-rich, systems between the energy source and the heat sink, with simpler forms taking on the initial interruption of the energy flow and shielding the evolution of more elaborate forms behind them. This in turn made it 
possible for even more delicate forms to evolve, and so on, with each succeeding level in the chain of energy dissipation using weaker gradients and pushing the energy further toward heat. To be sure, evolution increased the assimilation efficiency of the forms higher in the food chain, but, given the low energy efficiency of any effective material event, including the transfer of energy from one consumer to the next, these forms were still forced to search out rarer gradients and thus became animals.

\section{INFODYNAMICS}

Infodynamics (information dynamics) is a perspective that animates information theory by way of thermodynamics (Ulanowicz 1986, 1997, Brooks and Wiley 1988, Weber et al. 1989, Salthe 1993, 2000). Insofar as infodynamics is based on repeatable, knowable aspects of systems, I consider it basically a developmental perspective rather than an evolutionary one (see Salthe 1993). An alternative perspective on infodynamics that is oriented around evolution can be found in Brooks (1997). A fundamental postulate of infodynamics is that the formal isomorphism between Boltzmann's (1974) statistical interpretation of physical entropy as disorder and Shannon's formulation of variety as informational entropy (Shannon and Weaver 1949) signals a deep connection between information and entropy production. Because it is so general, the infodynamical perspective, which offers a nonequilibrial, process type of framework, can be applied to virtually any dynamic material system whatsoever.

This fact leads us to Fig. 1, a generalization of empirical curves taken from biological organisms (see especially Zotin 1972, Aoki 1991), ecosystems (see especially Aoki 1989, 2001, Jørgensen, 2001a,b), and several abiotic dissipative structures (Salthe 1989, 1993). In the spirit of the strategy of confirmation, which must always precede the strategy of testing, I postulate that these curves will hold for all natural dissipative structures, both biotic and abiotic. I know of no data that fail to corroborate this as yet. There are two major facts about these curves: (1) they begin by increasing and (2) they sooner or later start to slow down. We need to explain both of these phenomena, which together can be taken as Minot's law (Minot 1908, Needham 1964), or, indeed, because Minot referred only to organisms whereas Aoki recently tied all of this more widely together (Aoki 2001), the Minot-Aoki law.
The increasing function, when taken over growth in mass and/or growth in energy throughput (for other flows, see Ulanowicz 1986, 1997) or in embodied information (Jørgensen 2001b), seems perfectly general, and so calls for a general explanation. Why do dynamic things grow at all? And then, having started, why don't they just keep on growing, or even set off exponentially?

\section{The burgeoning}

Taking the first question first, today we have an answer from cosmology (Layzer, 1976, 1990, Frautschi 1982, 1988, Landsberg 1984; see also the connection to ecosystems in Jørgensen et al. 1998), which is that things grow because of the still accelerating universal expansion following the Big Bang. Dissipative structures grow for the same general reason that wave fronts spread and diffusion occurs, i.e., because the universe is way out of equilibrium and getting even more so all the time. Of course, more proximate causalities can be found for particular cases, but I am interested here in a very general understanding. Diffusion and the spreading of wave fronts serve the second law of thermodynamics by moving local situations toward equilibrium. Dissipative structures do the same by degrading energy gradients during growth and homeostasis in such a way as to produce entropy to an extent that is correlated with the rate at which they dissipate the gradients. That is, the faster a gradient is reduced, a proportionally smaller amount of its embodied energy can serve as exergy in the interests of that consumer, and a proportionally larger amount of it will head toward the sink in the direction of heat (Clausius 1851, Carnot 1976). The general situation is this: as the universal expansion accelerated beyond a certain rate, matter precipitated from, and is, energy that failed to stay in equilibrium. In its own haphazard search for equilibrium, matter collided and formed clumps, taking the universe even further from equilibrium. The universal response to this was to use some clumps to destroy others, and this project entrained the further evolution of complex structures all the way to living ones. This tactic works for the universe because, given the relatively poor energy efficiency of dissipative structures, more of an energy gradient must always be lost as heat than can become re-embodied in, or commanded by, its consumers.

Consequently, systems grow because they are linked to energy gradients in such a way that some of the energy, i.e., the exergy, in those gradients is 
transferred to these systems when they harness the resulting energy flow during their formation. Because the energy efficiency of effective natural systems is quite poor, so that as much or more of a gradient is lost than is assimilated, it can be said that systems grow to serve gradient degradation, which was in any case an historically prior process, thereby taking the second law to be a final cause of form and behavior. Extending this view hypothetically, we can surmise that energy degradation is especially attracted in the direction of being harnessed by growing systems, because that direction allows the fastest immediate breakdown of a gradient to a lower quality, however well or poorly it serves system growth. Of course, dissipation all the way to heat energy is the ultimate goal of the second law, but this can be delayed when one of the gradients in a cascade happens to become degraded relatively more rapidly by a process that leads into an overall slower line of second-law dissipation to heat than might have been found in some other pathway. The system, looking, as it were, backward rather than forward, may at any moment spontaneously take the most rapid immediate path to gradient dissipation, even if this eventually delays complete dissipation to heat. This general line of thought derives from Swenson (1989a, 1989b, 1997), Schneider and Kay (1994), and Matsuno and Swenson (1999). Swenson formulated a maximum entropy production principle to the effect that the universe everywhere acts to maximize entropy production, given local constraints. $\mathrm{He}$ and Schneider later simplified this to the principle that the universe acts to degrade all gradients as quickly as possible. The entropy production view is a global, externalist interpretation that is difficult to monitor, whereas the gradient dissipation version is a local, internalist one (Matsuno and Swenson 1999) that can be more readily measured. Because, even though it may be metastable, any particular energy gradient need not be dissipated, the entropy production view, by way of Boltzmann's interpretation, has the merit of providing a universal necessity for energy consumption.

Fig. 1. Idealized empirical curves of energy flow rates for dissipative structures (abiotic, ecological, and biological). The ordinate lists different variables that would be expected to behave as per the curves. Specific or intrinsic dynamics refer to rates per unit mass. The abscissa shows canonical stages of development as used in infodynamics. LotkaOdum maximization refers to the maximum power principle, which governs gross energy throughputs. This contributes the flow component to Ulanowicz's ascendency, which is an overall measure of development. The Prigogine minimum refers to the interpretation that Prigogine's minimum entropy production principle governs specific flows in the senescent stage.

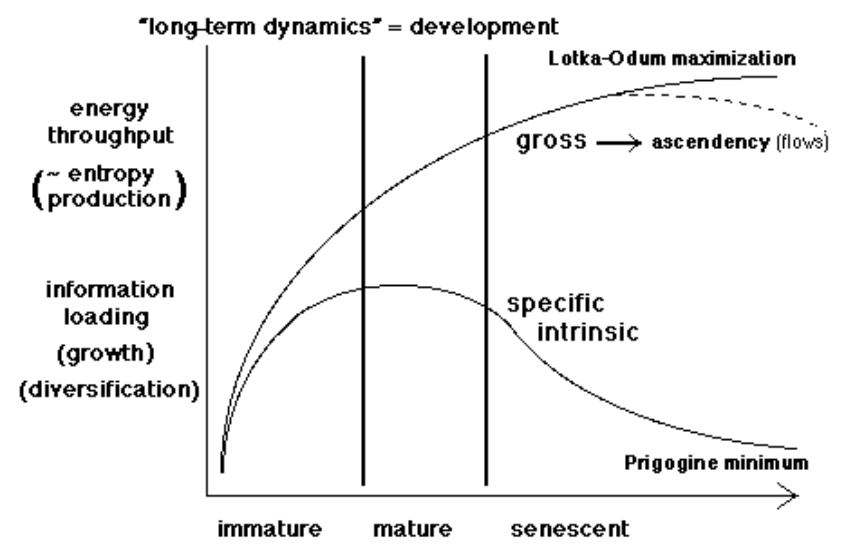

Systems grow relatively rapidly at first because they have not yet acquired any impediments to growth. Indeed, we may suppose that the rapid growth rates of immature systems best represent the universal urgency toward equilibration. However, we then notice a decline in the growth rates of older systems. There seems to be a law of diminishing returns for growth, and in ecological systems this is revealed as a limitation on diversification. I have proposed (Salthe 1993) that the limit to continued growth of any kind is imposed by information overload, because it is being loaded into a finite locale that is no longer growing. Information in this context is defined as any constraint on entropy production, and consequently any new twist in any configuration might, in principle, function as information. Constraints of this type are familiarly represented as constants in dynamical equations. Information overload works out as (1) overconnectedness, which leads to functional underconnectedness such as lags and delays, and (2) reinforcement of system propensities, which leads to a loss of flexibility in response to fluctuations. Both consequences have negative effects on energy throughput as well as on the requisite variety of the system (Ashby 1962, Conrad 1983), which then erodes its adaptability, setting it up for recycling.

Figure 1 shows that the Minot-Aoki law has had two well-known descendants: the Lotka-Odum maximum power principle (Lotka 1922, Odum and Pinkerton 1955) and the Prigogine minimum entropy production principle (Prigogine 1955), in which production 
diminishes to a steady state near system equilibrium. The interpretation given here (Salthe 1993) is that, because the gross rates in Fig. 1 frequently only level off even though the intrinsic (specific) rates continue to diminish, this allows us to hold onto the maximum power principle even in senescence in many systems. Nevertheless, some ecosystems do show declines, as indicated by the dotted line, which would require a special explanation. Note that the Prigogine principle is being interpreted (Salthe 1993) as a characteristic of senescence (see also Zotin 1972, Kay 1984, Jørgensen 2001a). The experiments that demonstrated it (Prigogine and Wiame 1946) developed spontaneously into this stable condition, which was then maintained by a continuing low-level energy input. Of course, natural dissipative systems do not get close to equilibrium at any stage, but the point is that in senescence they are heading in that direction. Environments are not nearly supportive enough to allow systems to even approach a truly steady state before being recycled.

Immature systems are characterized as relatively simple and are often relatively small, with a high and increasing intrinsic energy throughput that keeps them growing and self-transforming. They are generated by larger-scale systems at trivial energy cost, then need to hook up to significant energy sources to continue developing.

Senescent systems are becoming increasingly complex even though they are not growing in mass; they also become more metastable as their intrinsic energy throughput declines along with their flexibility, making it increasingly difficult for them to maintain themselves through adaptability in the face of perturbations.

Mature systems are in transition between the immature and senescent stages. In most kinds of natural systems they are so transient that they are indiscernible. Indeed, if it were not for the fact that biological systems, and the ecosystems co-opted by them, have gained unusual stability via genetic information, this stage would not need to be interpolated between the immature and senescent ones. In biological systems this is the stage at which reproduction occurs. Mature systems can be characterized as relatively complex and not growing, maintaining themselves in their less-thanmaximum complexity by way of a high gross energy throughput.
Fig. 2. Information theoretical curves for developing dissipative structures. The terms listed on the ordinate are different ways to express states of disorder, $H$, in words. $H_{\max }$ is the maximal behavioral variety a system could show given the kind of system it is, and includes nonfunctional and pathological states. $H_{\text {actual }}$ refers to the characteristic behavioral variety of a system, which is a restriction on $H_{\max }$ imposed by a system's organization. The distance between the curves roughly estimates a system's remaining developmental capacity. $H_{\text {actual }}$ is the measure of system complexity that contributes the storage component to Ulanowicz's ascendency. Resilience refers to the behavior of systems that experience rejuvenation when perturbed in the senescent stage.

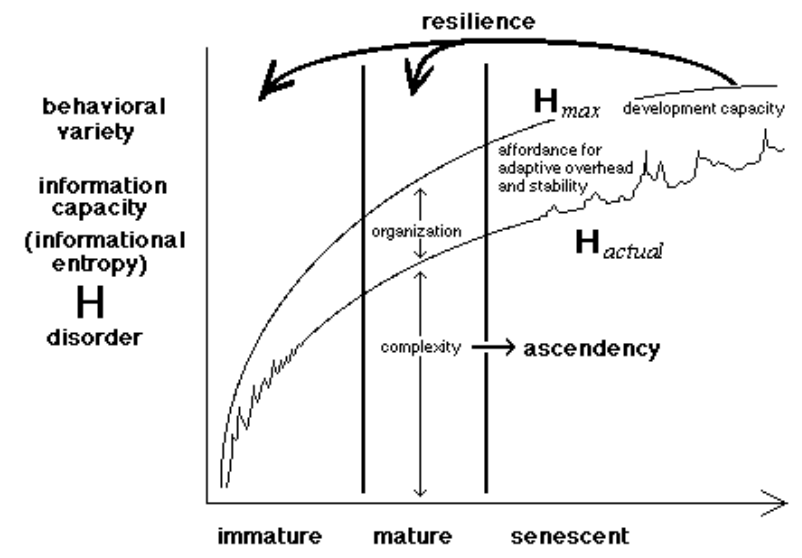

\section{The decline}

Figure 2 shows the informational entropy correlates of development, which are helpful in understanding the second fact about dissipative structures: that system growth and diversification do not continue indefinitely, but always eventually decline.

Once again, this formulation goes back to the cosmologists cited above, who wished to explain how form, i.e., order, information, could appear in the world despite the second law of thermodynamics. The answer they found was that, in a system expanding so fast that equilibrium is left behind, order could (I would prefer to say "must") increase along with entropy. Order, i.e., organization in Fig. 2, could be viewed as being just a consequence of a system that is not able to reach equilibrium, i.e., $H_{\max }$ in Fig. 2 (for cosmologists, $H$ or disorder would be replaced by $S$, physical entropy). The amount of order is evaluated as the distance between the curves. Furthermore, order would actually increase if the system continued to expand fast enough to cancel out the effects of equilibration. This increase would have to be at the 
expense of yet more entropy requirement, so that the curves in Fig. 2 must increasingly diverge as order, i.e., the distance between the curves, increases. The cosmological version of this figure would show only the two curves smoothly diverging (see Brooks and Wiley 1988).

As a result, the expanding universe is getting ever further from equilibrium as its burden of forms increases, making the second law ever more urgent along with continued complexity increase. For cosmology, complexity would be a label for the hierarchy of material forms, as in [galaxy [solar system [planet [some planetary macroscopic form]]]] (Salthe 1985). In general, complexity in the figure signifies organized complexity as in the scale hierarchy, whereas the distance between the curves represents disorganized complexity, a potential increase in behavioral possibilities that could be tapped to provide adaptability in emergencies. Consequently, the distance between the curves represents the imposition of system constraints on the variety of system behaviors, whereas fluctuations into this range from $H_{\text {actual }}$ show that unusual behaviors can be generated in emergencies.

Figure 2 has been made more complex than the original cosmological concept because it can now be proposed that these general relations apply to every individual dissipative structure inasmuch as these grow, which they do, either in size, numbers of components, and/or throughput. In this context, where we know much more about the details of system behavior, it is more fruitful to replace physical entropy with informational entropy. This can be done because Boltzmann's interpretation of physical entropy as disorder can be mapped onto Shannon's interpretation of variety as a form of entropy. Physical entropy is rooted in microscopic diffusion processes in which disorder increases as randomly moving particles access a greater variety of coordinates. If the occupied coordinates accessed by the particles could be used as digital tokens in some communication, we will have arrived at the Shannon concept, which may be taken as a macroscopic formulation or even a generalization (Tribus et al. 1966) of the same basic idea. It has been argued that, while the formal isomorphism holds, informational entropy need not increase if it changes, and so could not be a bona fide entropy. This view is falsified for any expanding or growing system, and I have argued (Salthe 1990) that informational entropy must grow as well (if it changes) when systems are modeled as from within. Even senescence does not curtail continued increases in embodied information, because all material systems continue to be marked by their experiences.

Next we need to briefly consider the idea of an ecosystem being taken as an individual. I have presented detailed arguments in support of this view in my book on scalar hierarchies (Salthe 1985), and so will not dwell on this vexed topic here. The major point is that, if our observations had the same scale relations to an organism as they have with respect to most ecosystems of biome size, we would not suppose an organism to be an individual either. It cannot be asserted that an ecosystem could not be an individual, and, if that idea appears useful for some serious reason, then individuality can provisionally be bestowed. Furthermore, there is no argument I know of that establishes individuality as an all-or-none category. In 1989 and 1993 I added to my argument for ecosystem individuality the fact that, when viewed from the very general perspective of infodynamics, there is sufficient evidence of the kind shown in Fig. 1 (see also Aoki 2001 and Jørgensen 2001a) to support this viewpoint, because these relations hold for organisms as well. This is the perspective, derived conceptually from E. P. Odum's 1969 paper (see also Schneider 1988 and Jørgensen 2001b) that I will bring to the facts considered below. Some moral considerations of this view were considered by Salthe and Salthe (1989). Note that I am not advocating ecosystems as "superorganisms" (Clements 1916, Wheeler 1928). Instead, I believe that, as seen from the present very general perspective, organisms could be taken for what one might call "superecosystems" (Depew and Weber 1995).

Turning again to Fig. 2, I note that information capacity would increase merely by way of system growth, e.g., increase in locales occupied, in numbers and kinds of components and/or processes such as cycling, and in possible global states. Growth generates informational constraints, i.e., symmetries or degrees of freedom, some of which will become lost, i.e., fixed, as information neat, during development as the system differentiates and individuates. The resulting information will generate an array of possible states that the system could occupy and display. Dynamic systems are active as well, exhibiting behavior globally and locally, both of which would increase, other things being equal, as the system acquires more structure. Consider the appropriateness of using the label "disorder" for the variety shown by macroscopic systems. In a more diverse ecosystem, an 
individual will face greater uncertainty as to its next encounter than in a more depauperate one. With order signifying regularity, the behavior of a species-rich system, which would generate many contingencies, would seem relatively disorderly at any locale, either for an internal player or for a naive external observer.

Why do dissipative structures grow in one way or another? The fundamental reason, noted above, is because they exist within an expanding, nonequilibrial universe. The final cause here is the need to produce entropy, which all growth leads to by way of derogating energy gradients in its service. However, each kind of system has its own material and efficient causes, i.e., its own peculiar connections to those gradients, which make up the basis of detailed discourse about the kinetics of each of them. What we can dwell upon here in more general terms are the formal causes, which we suppose to apply to all of them.

Given some kind of connection to a gradient and some configuration susceptible to efficient pushes, i.e., a location on the $H_{\text {actual }}$ curve in Fig. 2 determined by the variety of its behavioral repertoire and/or configurations, a dissipative system would be attracted by an array of possible resolutions concerning its next state, which would be somewhere between its own location and the one above it on the $H_{\max }$ curve. This upper curve, where the system would reach behavioral equilibrium, i.e., where it could access any potential behavior and state, functional or not, from any other, would represent the demise of the system, when it would have no further effective organized complexity. Its current embodiment alone prevents such a dash or explosion to instant extinction, restricting its reach to somewhere within its "adjacent possible" (Kauffman 2000) range. In other words, whatever slight material embodiment an immature system might have provides friction against its changes, which then are restricted to states that would produce somewhat more entropy than it currently does rather than totally dissipating its own embodied energy. Swenson $(1989 a, 1997)$ would say that a system changes in the direction of maximizing its entropy production; Schneider and Kay (1994) would say that it positions itself so as to maximize the rate of dissipation of its energy sources; Kauffman (2000) would say that it changes in such a way as to increase the size of its work surface; Jørgensen (1992, 1999, 2001a) would say that it chooses a developmental path that will maximize its exergy mobilization potential. These concepts have a large intersection that some would call a fourth law of thermodynamics. The upshot is that the system moves up on the $H_{\text {actual }}$ curve, accessing a somewhat greater range of behavioral variety, and so potentially imposing a greater entropy cost upon any observer or enemy engaging it.

Because this paper relates in particular to ecosystems, we should note a very general relation of the second law of thermodynamics to the growth of ecosystems (Taborsky 2000). If we examine energy gradient utilization quite closely, we will notice that not all that fails to be assimilated as exergy by a consumer is dissipated completely to heat. That is, dissipation in the sense of the second law, i.e., to fully disordered energy, is usually quite poor in most energy consumption. What actually happens is that much of any given gradient is dissipated into other gradients of much better quality than heat (recall TV scenes of sharks feeding). What we have in reality is a significant first-law contribution to dissipation, the result of which is to generate further gradients that can be used by other consumers. Gradient dissipation affords more gradient dissipation, and, in a sense, this fact must be the basis of all ecosystems. Of course, when these systems are permeated by living forms whose complexity is incompatible with the most rapid possible dissipation rates, dissipation all the way to heat occupies more time than it would need to with simpler consumers such as fires.

Ulanowicz (1986) has provided an autocatalytic cycle model of the formal causation of the increased complexification of system flows that works more closely into the kinetics, as opposed to the thermodynamics, of such a process. He constructs an overall quantitative measure of the degree of development of a system, referred to as its "ascendency," that combines flow rate increases (or growth in Fig. 1) with increases in the mutual information contained in the internal connections of the system, i.e., differentiation, which increases organization (see Fig. 2).

Note that in Fig. 2 the $H_{\text {actual }}$ curve shows considerable uncertainty as to its location in the immature range, which gradually damps out as the system increases its organization. The control by a very immature system over its range of behavior is limited because of its relatively limited amount of organization, but its homeorhetic recovery is strongly guaranteed by the centering gained from its tremendous energy flow per unit mass. 
So far we see that the $H_{\text {actual }}$ curve generates the $H_{\max }$ curve by, we might say, possible permutations of a system's controllable or functional behavior, which its embodiment largely restricts to some small search space (Brooks and Wiley 1988) wherein its developmental trajectory would be preserved (that is, as a Darwinian would say, if it survives at all). Simultaneously, we can see that the $H_{\text {actual }}$ curve is a material restriction on the $H_{\max }$ curve, which is ultimately determined by the kind of system in question, e.g., a ball bearing would have a much smaller absolute $H_{\max }$ than would, say, a nut (see the concept of "enformation" in Collier 1990). That is, $H_{\text {actual }}$ is in a sense carved out of $H_{\max }$ (Ulanowicz 1986, 1997). The space between them in this sense represents a reserve of unusual behavioral repertoire to be used in adapting to unusual fluctuations in the system's environment. Although this space was created by the information acquired by a system during its development, making it ever more informationally negentropic, access to this region would be an entropic behavioral excursion required by homeostasis. Such responses are always dangerous because the system moves closer to the $H_{\max }$ curve when using this reserve. Put another way (Ulanowicz 1986, 1997), this range between the curves represents the "overhead" wherein a system occasionally must pay extra entropy tribute for its continued existence.

Considering now the problem of why, in individual dissipative structures, the developmental process eventually terminates, we can recall that, in connection with Fig. 1, I suggested that this was a result of information overload. We can pursue this here again. The key feature in Fig. 2 is the increasing uncertainty of the position of the behavioral uncertainty curve $H_{\text {actual }}$ in the senescent stage. This signifies that the senescent system is becoming increasingly metastable: it experiences more frequent perturbations because of its relative rigidity and sluggishness of response and, in addition, has to reach more deeply into its entropic reserve behavior to recover from perturbations. As these fluctuations increase, the $H_{\text {actual }}$ curve gradually closes in on $H_{\max }$, which is no longer increasing very fast. Put in Ulanowicz's (1997:86-92) terms, the system is using up its developmental capacity, which, as scaled by system throughput, may actually decline. Eventually the $H_{\text {actual }}$ fluctuations get so close to $H_{\max }$ that the system collapses. In highly organized systems such as organisms, existence is terminated (note: after reproduction), and their component materials are recycled. In more loosely organized systems such as ecosystems, the system collapses back to some more immature condition, as in the figure-eight formulation of Holling (1978, 1986). Simulations (N. L. Johnson, unpublished manuscript) indicate that this rejuvenation is probably quite general in systems less highly organized than organisms. This system resilience is the tactic an ecosystem uses to escape from senescence and is comparable to the organism's tactic of reproduction and death. From an infodynamic point of view, death is preferable to senescence because the energy embodied in the system can be dissipated by detrivores more rapidly than it could itself manage to do with other gradients in its senescence. In much less organized abiotic systems such as tornadoes, system growth eventually disperses it so far from its focus on its energy gradient that it just falls apart, being dismembered, as it were, by other, more powerful dissipative structures. Once again, sluggish embodied energy is accelerated, one way or another, in the direction of equilibration.

\section{HUMAN-DOMINATED ECOSYSTEMS}

By the time humans come to actually dominate a locale, we will have perturbed it back to an early stage of development in the sense used above. The key to understanding this is human population growth, which under these conditions is usually accelerating. In advanced societies, human population growth is coupled to the steep energy gradients of farms and fisheries. Monocultures of annual plants are among the most immature of possible systems in any given locale, and in advanced societies their productivity is pushed along by the application of fertilizers. Fisheries rapidly deplete slow-reproducing, often large-bodied species, thus entraining the population growth of the more rapidly reproducing species (Weatherley 1972) so that, e.g., sardines replace tunnys. Of course, to the extent that it is growing, the human population is itself in an immature stage.

We must be careful, however, when reading the data. Infodynamics postulates that one form of ecosystem replaces another if the former produces more entropy per unit area and time and is also in a position to invade. This is often directly reflected in net primary production (NPP). However, as the map in Field (2001) shows, human appropriation in tropical regions has generally led to decreases in NPP. The reason, one supposes, is that most natural tropical terrestrial ecosystems cycle for the most part relatively quickly through the living components, with little storage in the soil. That is to say, natural mature and senescent tropical ecosystems cycle above the lateritic soils on 
which they stand. I would suggest that human appropriation here interferes with the system by removing key components, so that it senesces rapidly to a level set by the input of available energy, in a manner similar to those of Prigogine's experiments that led him to formulate the minimum entropy production principle. Equally plausible would be the idea that the system is held in a relatively underdeveloped state or edaphic climax (see below). The fact that even more temperate desert regions also show deficits of NPP after human takeover is presumably due to a similar disruption of highly evolved prior systems, which are replaced by new ones that cannot develop beyond some immature stage because harvesting prevents it. In some of these arid regions, we might note as well that human population growth is not at a level where it can entrain further entropy production either.

Note that, regardless of the direction of change in NPP, infodynamics predicts that all human-dominated systems produce more entropy than did prior natural ones, based on the fact of replacement itself. This, of course, would be linked to human activities, i.e., work, on behalf of these entrained systems, even though these activities may be funded from elsewhere.

In any case, a human-dominated ecosystem is more complicated than just a population and its food sources. Multitudes of other species of organisms will have been eliminated from the system, a key factor in making it more immature (even adding in our commensals, such as dandelions, starlings, and lice). Considering, for example, the concept of vegetation profile, the forests may be gone, but a humandominated system is not just dwellings and fields. As noted in papers given at the 100th Annual Meeting of the American Anthropological Association (Appendix 1) by J. R. Stepp (unpublished manuscript), J. T. McCabe (unpublished manuscript), and L. Pritchard Jr. (unpublished manuscript), information dominates human ecosystems. We need to note hedgerows, woodlots, roads and roadside berms, villages, parks, cemeteries, factories, storage depots, high-tension wire cuts, dams, and so on. If we consider a really advanced system with cities, then we have the equivalent of a vegetation profile in the facets and aspects of skylines and the like. It is an urgent project of infodynamics to find a way to calculate the relative amounts of information as constraints on entropy production in a city vs. a forest. It is clear that a city is more mature than a small village in these terms, including the size of its ecological footprint (T. Abel, unpublished manuscript), but we cannot yet compare it to a forest, which, in turn, however, we do know is more mature than a burn or an old field.

One factor in understanding a human-dominated ecosystem is that, discounting commensals, weeds, and pests, it has added another final cause to the need for entropy production, which still stands, of course, and is abetted by all of the above. This final cause is entrainment by population growth, which may well be maximized in most human-dominated systems, although not necessarily those where humans just barely fit in, such as in the Arctic or in some erstwhile tropical forests. All work is entrained by entropy production, as is all destruction; infodynamics supplies a theory of warfare, not to mention sports. From the infodynamic point of view, it can be postulated that humans came to dominate the world because the ecosystems we harness produce more entropy per unit time than any other form of ecosystem might in the same locales. Nevertheless, an old city is certainly a senescent system, sensu infodynamics. Of course, we have just the cure for this in warfare, which then rejuvenates a locale in the rebuilding. We have also instituted a kind of potlatch in the destruction of beautiful things like carpets and pottery, which makes some of them increasingly rare. As these artifacts are destroyed, the value of the remaining ones increaseshere, if anywhere, we can use Odum's (1983) idea of embodied energy (later, emergy)!

Perhaps the most important message of infodynamics is that stability in our systems is only metastability: we are ready at a moment's notice to pull the plug or push the button, initiating a new round of development. A less violent example of this principle is the business cycle (Soros 1998), which fits the development scheme of infodynamics quite well, demonstrating that capitalism is a growth ideology of immature systems, to which it returns again and again (as in iterations of Fig. 1).

\section{DISCUSSION}

Turning now to the present occasion of these thoughts, I will examine how some of the papers presented at the 100th Annual Meeting of the American Anthropology Association in Washington, D.C., on November 28December 2, 2001, (Appendix 1) under the rubric "Ecosystems and Complex Systems in Anthropology" can be frameworked by infodynamics in a "semiotic overview," as R. Zarger (unpublished manuscript) might say. On this head, I should reiterate that this 
effort, using Figs. 1 and 2, is a continuation of my practice of the strategy of confirmation in this direction (see also Salthe 2002 and in press). I do not at this juncture argue that this perspective is superior to any other for pragmatic reasons. Instead, I suggest that there is value in having a framework deeply rooted in cosmology that confers intelligibility upon multifarious data. I note in passing that infodynamics focuses intently on the "biophysical realities of the production process, especially thermodynamics," as called for by $\mathrm{C}$. Hall and R. Beal (unpublished manuscript).

The most obvious connections come through the papers of J. T. McCabe (unpublished manuscript) and J. Tainter et al. (unpublished manuscript). The former refers to "nonequilibrial" vs. "equilibrial" systems. The latter refers to "high-gain" systems (in terms of output per capita) that work steep energy gradients vs. "lowgain" systems that work shallow gradients, with a clear statement that the former tend to be supplanted by the latter over time. Nonequilibrial, high-gain systems are clearly immature in the infodynamic sense, whereas equilibrial (steady-state would be a better label), lowgain systems are relatively senescent. The table in Tainter et al. gives quite good characterizations of immature and senescent systems. A philosophical difference between these authors and myself is the issue of what, given remarks in another paper from this meeting (E. F. Moran, unpublished manuscript), we could call "reification." Infodynamics takes the phenomena in Fig. 1 to be a sign of system individuality. An individual need not be a highly autonomous system like an organism. For example, the business cycle (Soros 1998) shows this pattern, i.e., the Minot-Aoki Law, as does the simple arm movement of reaching. The latter starts out impulsively, then gradually refines towards the target, which, if it did not ease up, it would smash into. Put one way, this pattern is emergent, by which I mean that systems having very different material and efficient causes will come to evince it. Put another way, the pattern appears to be overdetermined, as by a structural attractor. The individuals in question here I have called "developmental trajectories" (Salthe 1993).

Developmental trajectories entrain material systems in the direction mandated by the second law of thermodynamics. Consequently, just like Hurricane Gloria, I myself am a material system entrained by an individual developmental trajectory of which I am at any given instant a momentary snapshot, similar to a view of, say, the stock exchange at the end of a given day with respect to one business cycle. The reason I see Tainter et al. as having a philosophical difference with this perspective is that they derive the pattern from an eventual external restriction on resources caused by overutilization by the system, which is defined as separate from its resources. This resource scarcity leads to renewed system growth as it searches for, and finds, new resources. C. Hall and R. Beal (unpublished manuscript) also believe that development is stimulated by resource depletion, that is, they seek what I would take to be a reductionist explanation of the transition from high- to low-gain systems, which they attribute to a reluctant switch by a system from steep to shallow gradients. I would seek instead to redefine the system to include immediate resources together with their consumers, which I take to change via development, providing that the result displays the pattern in Fig. 1. Along these lines, C. L. Redman (unpublished manuscript) sees development as being induced by "ecological drivers" that include geology, climatic variations, hydrology, and human activity. The infodynamics view places many of these drivers within the system, which then can be taken to be self-organizing. Note that this is no more alarming than seeing that biological cells have internalized their immediate energy gradients in the form of pyrophosphates.

Of course, this switching upscale needs to be done judiciously, with an eye to the problems that one has in mind. Formally, one could switch from a given biome to the solar system itself, given that the energy source of most ecosystems is ultimately the sun. It might even be possible to view the solar system as self-organizing, and I for one am willing to bet that it would show the pattern in Fig. 1, but this is going a bit far, one supposes, in terms of ecology. That is to say, I am switching upscale only to the degree needed to characterize a system as a self-organizing individual trajectory. Put another way, it turns out that I am assuming that most ecosystems can be taken to be individuals somewhat in the manner of Clements (1916), whose approach was also based on development, i.e., ecological succession. Again, I am not supporting the much stronger notion of ecosystems as kinds of organisms, but rather the reverse. I am basing this view on the fact that, when seen from the most general infodynamic perspective, individuals of different kinds exhibit the same basic phenomena.

No approach is without problems when it comes to harnessing actual data to it, and J. T. McCabe's (unpublished manuscript) study of the Turkana herders 
is an instructive case in point. These herders do not fit neatly into any developmental stage. However, this does not mean that nothing of interest can be derived from the developmental perspective. In fact, this type of apparent incoherence often signals that something is about to be learned. I would place the Turkana system at a point between senescent and immature, held between the end of one and the beginning of another developmental trajectory by the extreme poverty of the arid system they are part of. The growth of their system is stunted, but, like any immature system, it could begin growing at any moment. In addition, their regime is density-independent, because at any time their resources are unsaturated. So far their system is immature but ready to develop, given the chance. At the same time, it shows the large stochastic effects of a senescent system responding to perturbations (see the fluctuations of $H_{\text {actual }}$ in the senescent region of Fig. 2), in connection with which the Turkana have a very flexible opportunistic repertoire (see the fluctuations of $H_{\text {actual }}$ in the immature region of Fig. 2). I take the Turkana system to be underdeveloped in the infodynamic sense, and it could be said to be part of an edaphic climax. However, this situation does not really provide an opportunity for development, as a capitalist thinker might suppose; rather, I take it to be the best way to generate any entropy at all in an arid setting. This example shows that the canonical developmental pattern (Figs. 1 and 2) should be used as an analytical tool rather than an imposed reification. It could be fully realized only in a world without historical entanglements and contingencies.

Another example that seems at first problematic is the island of Bonaire (T. Abel, unpublished manuscript). Here there is an embarrassment of riches. The place is highly developed, i.e., no longer developing, with, presumably, a fairly high gross energy throughput. It is also very efficient in export/import terms $(=1.26)$. These are all senescent characteristics. Despite the fact that the place is so small and has no resources to speak of, the classification of senescence is reinforced by noting Bonaire's large "ecological footprint," as though it had grown in search of resources in the manner discussed by Tainter et al. (unpublished manuscript). However, it is not self-sustaining in the manner of a well-developed mature system, but instead is propped up from outside by the global capitalist system, of which, indeed, it is just a piece. That global system itself will presumably remain immature as long as fossil fuels are cheap, and Bonaire could then continue to be held in a long-sustained senescence.
Several of the papers considered here emphasize that human-dominated ecosystems like that of Bonaire, i.e., systems where we construct a "human appropriation of primary productivity" (H. Haberl and F. Krausmann, unpublished manuscript), become dominated by, as it were, "extra" information, from "people, politics, and information" (L. Pritchard Jr., unpublished manuscript). Following Lemke (1995), I refer to such systems as ecosocial systems. For example, J. T. McCabe (unpublished manuscript) found that a significant number of the perturbations of the unstable Turkana system were initiated by political fluctuations rather than biophysical ones. C. L. Redman (unpublished manuscript) formally proposes that political economy has as much influence on human ecology as do local ecosystemic processes, whereas E. F. Moran (unpublished manuscript) goes so far as to suggest that social and economic uncertainties have a greater effect on the fluctuations of ecosocial systems than do biophysical uncertainties. Furthermore, J. R. Stepp (unpublished manuscript) notes that some of these systems even come to dominated by a "continued persistence of maladaptive cultural processes."

Of course, all ecosystems involve "interactive flows of energy, matter, and information" (S. E. van der Leeuw, unpublished manuscript), as reflected in Figs. 1 and 2 above. In infodynamics, information is defined in a very general sense as constraints on entropy production, that is, whatever configurations interrupt the flow, sensu Prigogine (1955), of entropy from energy gradient dissipations are informational. The result of these interruptions is to increase the completeness of dissipation, in the strict second-law sense of dissipation all the way to heat. For example, a series of rock ledges deflecting a stream that is dissipating gravitational energy is imposing geological information upon that process. Generally, such information creates friction against the flow of, in this case, dissipated solar energy, thereby producing heat and, of course, is itself gradually eroded in the process. Living systems do the same, channeling the energy transformations into fine tessellations, in each of which entropy is produced from increasingly lowergrade, i.e., moderated, gradients. The connection here with biological genetic configurations again points up the fact that all information is historical in nature, and therefore might have been different. Anything that might have been different is information, providing that it delays energy dissipation to the effect of dissipating it more completely.

Because information delays the local flow of entropy 
to the sink, we need to determine whether or not this is a defiance of the second law. In the big picture, it is not, because information is a transformation of the energy embodied in matter that itself needs to be dissipated, and this it gradually is via frictions of one kind or another if it functions as information. In other words, those rock ledges are eventually ground down to substances more easily dissolved, conducted, and diffused. Furthermore, life was favored even though it delays the flow of entropy from the sun to the sink in space, because its agency drags matter out of the earth, putting it too in the way of erosion to forms that are more easily dissipated. Life also degrades the energy it taps further in the direction of heat than would have been the case had the energy not been captured in life's meshes. The idea is that information, including living information, succeeds in persisting because it increases the effectiveness of energy dissipation to its lowest grade (heat) accessible locally. Although entropy production internally in living systems is controlled to the point of being less than it might be for the same energy use in less organized systems, this is more than balanced by the entropy produced externally from the gradients that living systems consume (Schroedinger 1956, Collier 1988). Consequently, life is the universe's way of dissipating tightly closeted energy gradients that are not accessible to cruder erosions, and of dissipating it to quite a low grade in the process.

As it happens, human-dominated ecosystems have generally been simplified in the interests of increasing the energy flows within them, although this is accomplished at the cost of not obtaining as complete a dissipation as would have normally been the case in the more mature ecosystems they displaced. In this sense, human exploitation imposes a deficit of information on the transformations of solar energy and, as a result, generates more wastes of a higher grade than heat, which often end up acting as pollution. Human systems succeed in commandeering the energy gradients in different locales because they harness more powerful energy flows than do natural systems of the same overall scale. However, as noted above, ecosocial systems are more than just pastures, farms, and fisheries. Much of the energy captured in primary productivity is harnessed by political and economic informational arrays to produce the more or less elaborate superstructures and behavioral displays of ecosocial systems. This large array of informational channels for energy flow will generate further constraints on adjacent ecosystems as these are gradually brought under domination by serving as the recipients of, and being entrained by, relatively high-grade wastes.
Of course, even superstructures eventually begin to senesce, which requires that energy be expended on repairs. It is rare, however, for these forms to survive very long, because considerable energy flow is typically deflected toward warfare. War results in the episodic erasure of elaborate superstructures, which can then harness renewed energy flows in reconstruction, leading to the sort of boom and bust cycle typical of business in the capitalist system. With the Turkana too (J. T. McCabe, unpublished manuscript), warfare seems to stimulate renewed attempts at development that take the form of switching to new pastures.

It is an open question whether human-dominated ecosystems typically degrade more of the available energy gradients overall than would a more "natural" successional sere over the same period of time. The viewpoint espoused here proposes that they would and, indeed, that they could not have spread as they have if they did not. Here we need to distinguish again between first- and second-law dissipations. First-law dissipation is into many different energy gradients of a higher grade than heat, whereas second-law dissipation is all the way to heat. Although the powerful energy transformations of immature systems may rapidly dissipate most of a gradient, much of it is transformed into "wastes" of a higher grade than heat, whereas the more moderate dissipations of more mature systems would over the same period take less total gradient but go further toward heat with it. The grand ecosocial systems we know about, and our own, seem entrained more into first-law dissipation, deploying the more powerful resulting energy flows against the rest of the world.

Weaker dissipative structures are sooner or later destroyed by, or incorporated into, more powerful ones adjacent to them. The latter frequently lay waste to the former. More immature, stronger energy flows, guided as they are by fewer informational constraints, produce more high-grade waste than do weaker, more mature ones, and these wastes, as is now well known, tend to rejuvenate adjacent ecosystems as well. Of course, the ultimate immature system is a desert, where the sun's energy is mostly just reradiated into the energy sink and hindered only by windforms it drives. Although these energy flows are maximal, they generate relatively little further entropy in the friction that accompanies flying sands, which produce only gradual surface erosion. This represents a major setback to the universal project of entropy production, which is exactly why, in the infodynamic view, life would be 
favored wherever possible. On Earth, desertified residues of human activity persist in producing as much entropy as possible, giving us various partially desertified ecosystems, as in the Mediterranean landscapes (S. E. van der Leeuw, unpublished manuscript) or the lands of the Turkana (J. T. McCabe, unpublished manuscript).

We can now address the question of whether humandominated ecosystems typically impose less, or as much, or more information on energy flows than do other ecosystems. As noted, if human-dominated ecosystems generate such powerful energy flows that they can incorporate or destroy other ecosystems and show relatively poor second-law dissipation as well, then they must be imposing fewer overall informational controls on energy flows, which is a sign of a relatively immature configuration. Here again warfare has been important. If ecosocial systems were to develop into truly senescent systems, their overall energy throughput would become more moderate, shedding fewer high-grade wastes. However, intermittent warfare has derailed this tendency, with the result that development is typically curtailed beyond the point at which an ecosocial system would no longer be powerful enough to co-opt and appropriate other ecosystems. Consequently, when it is suggested that human ecosystems are informed as much by ecosocial informational arrays as they are by "natural" ones (J. T. McCabe, unpublished manuscript; E. F. Moran, unpublished manuscript; L. Pritchard Jr., unpublished manuscript; J. R. Stepp, unpublished manuscript), this must mean, in my view, that, because human institutional information does not fully make up in amount for the information it has displaced, there would typically be as much a loss of information as a co-option when humanity appropriates an ecosystem.

Let me take this opportunity to derail what is probably a growing suspicion in the minds of some readers. Is infodynamics just another totalizing reductionist program that puts too much emphasis on calorie counting? No, because it is not a full program of understanding at all. It is a program aimed at understanding complex systems from a quite low integrative level, i.e., the material, from the intensional complexity perspective (Salthe 1993), as in: [physical world [material world [biological world [cognitivetechnological world]]]]. In the specification hierarchy \{work entrained by entropy production \{ material order accumulation \{biological organization building \{human ecosocial institution building \}\}\}, we can see that infodynamics, as deployed herein, deals only with the lowest of these levels. It need not be in conflict with more specified theories deployed at other levels of integration, provided that they do not abrogate or transcend the constraints imposed at the lower levels. For example, if a human culture existed that cultivated a senescent lifestyle, and surely at least one isolated one must have done so for a while, infodynamics makes it possible to place this culture thermodynamically but would not necessarily facilitate an understanding of its origin, as opposed to its natural affordances, or of the details of how it was played out. Infodynamics is necessary but insufficient for understanding ecosystems. It predicts the demise of any senescent system, but not how or when this will be brought about historically, just as each economic bust has its own unique history.

Finally, we should consider briefly the need expressed by several of the authors who presented the papers listed in Appendix 1 (T. A. Kohler, unpublished manuscript; J. T. McCabe, unpublished manuscript; E. F. Moran, unpublished manuscript; C. L. Redman, unpublished manuscript) to incorporate human agency, otherwise known as "path dependence" or historicity, into models of ecosocial systems. This is an example of an important aspect of things that infodynamics has little to say about, other than the following thought experiment. Consider a township, e.g., the placing of houses, woodlots and fences; the exact location and boundaries of roads and villages. Now suppose we have access to multiple worlds, from which we can pick out any number of other counterfactual townships in the same location, each of which would have had a different history. Family names and famous folks could all be different. Even the locations of old trees could be different, but climate and weather would be about the same for all. Infodynamics makes only one prediction about the ensemble of alternate versions: that the cumulative entropy production of most of them will be within a standard deviation of the mean for the collection.

\section{CONCLUSIONS}

I share with several of the authors who presented papers from the list in Appendix 1 (H. Haberl and F. Krausman, unpublished manuscript; C. Hall and R. Beal, unpublished manuscript; J. Tainter et al., unpublished manuscript) an interest in ecological sustainability. However, I strongly agree that the term "sustainable development" is oxymoronic (C. Hall and R. Beal, unpublished manuscript). Infodynamically, growth occurs in the immature stages of a system's 
development, whereas sustainability is possible only from maturity onwards, although the likelihood of achieving it diminishes as a system continues to senesce. Of course, sustainability may mean different things to different people. Some might consider the boom and bust of the business cycle a kind of resilience (Holling 1976) as in Fig. 2, which ought to be sustained as long as possible. Of course, those who lose their jobs in a bust might not be able to make such a cool assessment. Others might see sustainability in the core nations of a global economic system while growth continues in the periphery. However, this setup must eventually senesce globally, as in the present case, when cheap sources of energy are exhausted or when all lands with cheap labor have been colonized, whichever occurs first. C. Hall and R. Beal (unpublished manuscript) note that the notion of sustained development emanates from neoclassical economics, which is not rooted in natural science.

My own recommendation for sustainability, rooted in infodynamics, would be for a system to try to preserve infodynamic maturity for as long as possible. This goal would, however, entail a good deal of planning, as can be seen in a simple example. We all know firsthand the pain of roadwork. Roads do senesce, but the pain could be alleviated with careful planning that involved balancing road materials with expected, and enforced, usage rules; evaluating weather conditions; taking into account greater than allowable usage; repairing widely scattered short sections at a time; investing in research on self-healing road materials; and so on. The reason why none of this is typically done at present comes down, of course, to the growth ideology of capitalism, probably in more than one form, i.e., the profitability of totally remaking the roads, including the jobs involved, as well as graft, unplanned and unexpected growth, etc. Our society would need first to be convinced that the growth ideology has too many downsides, such as depressions and wars, something that seems unlikely until the ultimate decline of cheap per joule energy sources. Why unlikely? Because the second law of thermodynamics is the most powerful of the final causes that dominate our activities.

Responses to this article can be read online at: http://www.consecol.org/vol7/iss3/art3/responses/index.html

\section{Acknowledgments:}

I would like to thank Ichiro Aoki and Jack Maze for comments on the introduction, and John Collier and Bob Ulanowicz for comments throughout.

\section{APPENDIX 1}

This study refers to the findings of 12 papers presented at the 100th Annual Meeting of the American Anthropological Association, Washington, D.C., November 28-December 2, 2001. At the time of writing, the proceedings had not yet been published, so these papers are cited in the text as unpublished manuscripts. Full author and title information is provided here for readers who may be interested in pursuing them further.

Abel, T. 2001. Human ecosystems, self-organization, and emergent hierarchy: economic growth and the case of ecotourism on Bonaire.

Haberl, H., and F. Krausmann. 2001. The energetic metabolism of societies and human appropriation of net primary production (HANPP); empirical examples for Austria 1830-1995 on different spatial levels.

Hall, C., and R. Beal. 2001. Neoclassical economics, biophysical economics and the myth of sustainable development.

Kohler, T. A. 2001. Agent-based modeling and ecosystem anthropology.

McCabe, J. T. 2001. Ecosystems at disequilibrium and Turkana mobility.

Moran, E. F. 2001. Global environmental change and the ecosystem perspective.

Pritchard, L., Jr. 2001. Ecological surprise and the emergence of adaptive capacity: lessons from ecological- 
economic-social linked systems.

Redman, C. L. 2001. Historical legacies, patterns, and insights as a key component of modern ecological studies.

Stepp, J. R. 2001. Unique properties of human ecosystems.

Tainter, J., T. F. H. Allen, and T. W. Hoekstra. 2001. Sustainabily and resource transitions.

van der Leeuw, S. E. 2001. The role of information flows in dynamically structuring society.

Zarger, R. 2001. Graphical representations as phenomenon and tool in understanding human ecosystems.

\section{LITERATURE CITED}

Aoki, I. 1989. Holological study of lakes from an entropy viewpoint-Lake Mendota. Ecological Modelling 45:81-93.

Aoki, I. 1991. Entropy principle for human development, growth and aging. Journal of Theoretical Biology 150:215223.

Aoki, I. 2001. Entropy and the exergy principles in living systems. Pages 167-190 in S. E. Jørgensen, editor. Thermodynamics and ecological modelling. Lewis, Boca Raton, Florida, USA.

Ashby, C. R. 1962. Requisite variety, and its implications for the control of complex systems. General Systems Yearbook 9:99-105.

Boltzmann, L. 1974. The second law of thermodynamics. Pages 1-8 in B. McGuinness, editor. Theoretical physics and philosophical problems. D. Reidel, Dordrecht, The Netherlands.

Brooks, D. R. 1997. Biological evolution as a microcosm of cosmological evolution. Bridges 4:9-35.

Brooks, D. R., and E. O. Wiley. 1988. Evolution as entropy: towards a unified theory of biology. University of Chicago Press, Chicago, Illinois, USA.

Carnot, S. 1976. Reflections on the motive power of fire, and on machines fitted to develop that power. Pages 16-35 in J. Kestin, editor. The second law of thermodynamics. Dowden, Hutchinson, and Ross, Stroudsburg, PA, USA.

Clausius, R. 1851. On the moving force of heat and the laws regarding the nature of heat itself which are deducible therefrom. Philosophical Magazine (Series 4) 2:1-12.

Clements, F. E. 1916. Plant succession: an analysis of the development of vegetation. Publications of the Carnegie Institution of Washington, D.C., Publication Number 242. Carnegie Institution, Washington, D.C., USA.

Collier, J. D. 1988. The dynamics of biological order.
Pages 227-242 in B. H. Weber, D. J. Depew, and J. D. Smith, editors. Entropy, information, and evolution: new perspectives on physical and biological evolution. MIT Press, Cambridge, Massachusetts, USA.

Collier, J. D. 1990. Intrinsic information. Pages 390-401 in P. P. Hanson, editor. Information, language, and cognition. Oxford University Press, New York, New York, USA.

Conrad, M. 1983. Adaptability: the significance of variability from molecule to ecosystem. Plenum Press, New York, New York, USA.

Depew, D. J., and B. H. Weber. 1995. Darwinism evolving: systems dynamics and the genealogy of natural selection. MIT Press, Cambridge, Massachusetts, USA.

Field, C. B. 2001. Sharing the garden. Science 294:24902491.

Frautschi, S. 1982. Entropy in an expanding universe. Science 217: 593-599.

Frautschi, S. 1988. Entropy in an expanding universe. Pages 11-22 in B. H. Weber, D. J. Depew, and J. D. Smith, editors. Entropy, information and evolution. MIT Press, Cambridge, Massachusetts, USA.

Holling, C. S. 1973. Resilience and stability of ecological systems. Annual Review of Systematics and Ecology 4:1-23.

Holling, C. S. 1986. The resilience of terrestrial ecosystems. Pages 292-320 in W. C. Clark and R. E. Munn, editors. Sustainable development of the biosphere. Cambridge University Press, Cambridge, UK.

Jørgensen, S. E. 1992. Development of models able to account for changes in species composition. Ecological Modelling 62:195-208.

Jørgensen, S. E. 1999. State-of-the-art of ecological modelling with emphasis on development of structural dynamic models. Ecological Modelling 120: 75-96.

Jørgensen, S. E. 2001a. A tentative fourth law of 
thermodynamics. Pages 303-348 in S. E. Jørgensen, editor. Thermodynamics and ecological modelling. Lewis, Boca Raton, Florida, USA.

Jørgensen, S. E. 2001b. Toward a consistent pattern of ecosystem theories. The Scientific World 1:71-75.

Jørgensen, S. E., H. Mejer, S. N. Nielsen, and J. Teuber. 1998. The evolution of the thermodynamic equilibrium in the expanding universe. Physica Scripta 58:543-544.

Kauffman, S. A. 2000. Investigations. Oxford University Press, New York, New York, USA.

Kay, J. J. 1984. Self-organization in living systems. Dissertation, University of Waterloo, Waterloo, Canada.

Landsberg, P. T. 1984. Can entropy and 'order' increase together? Physics Letters 102A:171-173.

Layzer, D. 1976. The arrow of time. Scientific American 233:56-69.

Layzer, D. 1990. Cosmogenesis. Oxford University Press, New York, New York, USA.

Lemke, J. L. 1995. Textual politics: discourse and social dynamics. Taylor and Francis, London, UK.

Lotka, A. J. 1922. Contribution to the energetics of evolution. Proceedings of the National Academy of Science 8: $147-151$.

Matsuno, K., and R. Swenson. 1999. Thermodynamics in the present progressive mode and its role in the context of the origin of life. BioSystems 51:53-61.

Minot, C. S. 1908. The problem of age, growth and death. Murray, London, UK.

Needham, A. E. 1964. The growth process of animals. Pitman, London, UK.

Odum, E. P. 1969. The strategy of ecosystem development. Science 164:262-270.

Odum, H. T. 1983. Systems ecology: an introduction. Wiley Interscience, New York, New York, USA.

Odum, H. T., and R. C. Pinkerton. 1955. Time's speed regulator, the optimum efficiency for maximum output in physical and biological systems. American Scientist 43:331343.

Prigogine, I. 1955. Introduction to thermodynamics of irreversible processes. Interscience, New York, New York, USA.

Prigogine, I., and J. M. Wiame. 1946. Biologie et thermodynamique des phénomènes irréversibles. Experientia 2:451-453.

Salthe, S. N. 1985. Evolving hierarchical systems: their structure and representation. Columbia University Press, New York, New York, USA.

Salthe, S. N. 1989. Self-organization of/in hierarchically structured systems. Systems Research 6:199-208.

Salthe, S. N. 1990. Sketch of a logical demonstration that the global information capacity of a macroscopic system must behave entropically when viewed internally. Journal of Ideas 1:51-56.

Salthe, S. N. 1993. Development and evolution: complexity and change in biology. MIT Press, Cambridge, Massachusetts, USA.

Salthe, S. N. 2000. Ecology and infodynamics. Journal of Social and Evolutionary Systems 21:223-231.

Salthe, S. N. 2002. An exercise in the natural philosophy of ecology. Ecological Modelling 158:167-179.

Salthe, S. N. In press. The natural philosophy of ecology: developmental systems ecology (infodynamics). In J. Drake and C. Zimmerman, editors. Complexity in ecological systems. Columbia University Press, New York, New York, USA.

Salthe, S. N., and B. M. Salthe. 1989. Ecosystem moral considerability: a reply to Cahen. Environmental Ethics 11:355-361.

Schneider, E. D. 1988. Thermodynamics, ecological succession and natural selection: a common thread. Pages 107-138 in B. H. Weber, D. J. Depew, and J. D. Smith, editors. Entropy, information and evolution: new perspectives on physical and biological evolution. MIT Press, Cambridge, Massachusetts, USA.

Schneider, E. D., and J. J. Kay. 1994. Life as a manifestation of the second law of thermodynamics. Mathematical and Computer Modelling 19:25-48.

Schroedinger, E. 1956. What is life? and other scientific essays. Doubleday Anchor, Garden City, New York, USA.

Shannon, C. E., and W. Weaver. 1949. The mathematical theory of communication. University of Illinois Press, Urbana, Illinois, USA.

Soros, G. 1998. The crisis of global capitalism (open society endangered). Little, Brown, London, UK.

Swenson, R. 1989a. Emergent attractors and the law of maximum entropy production: foundations to a theory of general evolution. Systems Research 6:187-198.

Swenson, R. $1989 b$. The earth as an incommensurate field at the geo-cosmic interface: fundamentals to a theory of emergent evolution. Pages 19-22 in Proceedings of the First Congress on Geocosmic Relations. PUDOC Science, Amsterdam, The Netherlands. 
Swenson, R. 1997. Autocatakinetics, evolution, and the law of maximum entropy production. Advances in Human Ecology 6:1-47.

Taborsky, E. 2000. The complex information process. Entropy 2: 81-97.

Tribus, M., P. T. Shannon, and R. B. Evans. 1966. Why thermodynamics is a logical consequence of information theory. Journal of the American Institute of Chemical Engineering 12:244-248.

Ulanowicz, R. E. 1986. Growth and development: ecosystems phenomenology. Springer-Verlag, New York, New York, USA.

Ulanowicz, R. E. 1997. Ecology, the ascendent perspective. Columbia University Press, New York, New York, USA.

Weatherley, A. H. 1972. Growth and ecology of fish populations. Academic Press, London, UK.

Weber, B. H., D. J. Depew, C. Dyke, S. N. Salthe, E. D. Schneider, R. E, Ulanowicz, and J. S. Wicken. 1989. Evolution in thermodynamic perspective: an ecological approach. Biology and Philosophy 4:373-405.

Wheeler, W. M. 1928. Emergent evolution and the development of societies. Norton, New York, New York, USA.

Zotin, A. I. 1972. Thermodynamic aspects of developmental biology. Karger, Basel, Switzerland. 